\title{
Procedimiento de investigación de casos de enfermedades profesionales
}

\author{
Jerónimo Maqueda Blasco y José María Roel Valdés
}

DOI: 10.12961/aprl.2019.22.04.01

A pesar de que la contribución de la enfermedad profesional o relacionada con el trabajo a la carga de morbilidad de las poblaciones trabajadoras se estima muy superior a la contribución del accidente de trabajo ${ }^{1}$, llama la atención el escaso desarrollo de una práctica de investigación orientada a la identificación de los hechos causales.

La ocurrencia del accidente de trabajo como un hecho brusco, disruptivo de la normalidad en los procesos de trabajo y siempre dramático en sus consecuencias, genera una reacción inmediata de necesidad de intervención tanto desde las estructuras preventivas de las empresas como desde las Autoridades Laborales, esto deriva en la puesta en marcha de procedimientos de investigación de campo.

La enfermedad profesional o relacionada con el trabajo tiene un comportamiento epidemiológico claramente diferente $y$, además, de amplia diversidad. La enfermedad es un suceso preventivamente "incómodo" y fácilmente deslizable hacía ámbitos explicativos fuera de los entornos de trabajo. Lo que conduce a que, finalmente, no se aborde su investigación.

Existen factores concurrentes que favorecen este escenario, como pueden ser, entre otros, la debilidad de nuestro sistema preventivo en las especialidades de Medicina y Enfermería del Trabajo, situación puesta de manifiesto en el informe de la Comisión Nacional de Seguridad y Salud en el Trabajo ${ }^{2}$, el escaso desarrollo de la vigilancia de la salud colectiva, la ausencia de procedimientos armonizados de investigación, etc.

La preocupación social por cómo se está afrontando el tema de las enfermedades relacionadas con el trabajo y particularmente las enfermedades profesionales, ha quedado reflejada en la Estrategia Española de Seguridad y Salud en el Trabajo (EESST) 2015-202033, cuyo objetivo 3.B expresa la necesidad de fortalecer las acciones dirigidas a una mejor identificación de las causas, efectos y medidas de prevención de la enfermedad profesional. Este objetivo no deja de ser una llamada para llegar, a partir de la investigación del caso, a un conocimiento colectivo que permita dirigir de forma selectiva las medidas de prevención y por lo tanto mejorar su propia efectividad.

a. Instituto Nacional de Seguridad y Salud en el Trabajo, Madrid, España.

b. Universidad Miguel Hernández, Alicante, España.
La identificación de las causas de la enfermedad tiene su base en los métodos de Epidemiología Aplicada de Campo que permiten responder a los interrogantes de: ¿Qué? (naturaleza de la enfermedad), ¿Quién? (características y actividad de la persona afectada), ¿Dónde? (características del lugar de trabajo y su entorno). ¿Cuándo? (momento temporal en el que se manifiesta la enfermedad), ¿Cómo? (mecanismos, vías o formas de contacto de la persona con el agente potencialmente causante de la enfermedad) y ¿Por qué? (agente, condiciones o causas determinantes de la aparición de la enfermedad y su origen).

La investigación de la enfermedad profesional presenta hechos diferenciales con respecto a la investigación del accidente de trabajo, siendo el hecho más crítico el establecimiento de las relaciones causa/efecto, lo que depende de: (i) la naturaleza del agente y del tiempo e intensidad de la exposición, (ii) la forma de prestación de la enfermedad (aguda, subaguda o crónica) y (iii) la variabilidad en la respuesta bio-médica de la persona expuesta.

Todo ello genera periodos de latencia, en ocasiones lo suficientemente prolongados para establecer la asociación causa/ efecto que buscamos.

Por otra parte, el comportamiento epidemiológico de la enfermedad tiende a formar agregados de casos, como por ejemplo los eccemas alérgicos de contacto, las alveolitis alérgicas extrínsecas, etc.

La complejidad de la investigación de la enfermedad profesional hace que el establecimiento de un procedimiento armonizado de investigación sea especialmente necesario.

La utilidad de un procedimiento de investigación de casos de enfermedades profesionales ha sido demostrada en 2013 por Roel Valdés J.M. ${ }^{4}$, en el ensayo de un procedimiento de investigación aplicado a 21 casos incidentes de enfermedades profesionales. Los 13 investigadores de ámbitos de servicios de prevención tanto ajenos como propios, que aplicaron el procedimiento valoraron con un acuerdo de Excelente (100\%-90\%) o Alto (89\%-70\%) las dimensiones relativas a Necesidad, Suficiencia y Utilidad.

Con estos antecedentes el Instituto Nacional de Seguridad y Salud en el Trabajo (INSST) abordó el reto de poner a disposición de los Servicios de Prevención un procedimiento de investigación de casos de enfermedades profesionales y relacionadas con el trabajo ${ }^{5}$, que si bien tiene como destinatarios principales a los Servicios de Prevención, su uso puede ser de utilidad para otros profesionales que afronten la investigación de este tipo de enfermedades. 
El principal valor de una investigación de la enfermedad profesional de calidad es el contribuir a garantizar la equidad hacia el trabajador que contrae una enfermedad en la investigación de su origen, naturaleza y medidas preventivas. Así mismo, las acciones comprendidas en la investigación permiten: (i) Identificar los hechos causales asociados con la aparición de la enfermedad profesional o relacionada con el trabajo y (ii) Afrontar la investigación de casos de enfermedades profesionales o relacionadas con el trabajo de una forma estructurada y armonizada.

La investigación del caso se afronta desde una perspectiva multidisciplinar, lo que implica la necesidad de establecer un equipo de investigación que desarrolle sus trabajos en torno a tres ejes de actuación: (i) entrevista médico-laboral con el trabajador, (ii) análisis de la documentación preventiva e (iii) información obtenida de la observación directa de las condiciones de trabajo.

Estos ejes se instrumentalizan mediante dos herramientas aplicadas: una dirigida a la sistematización de la recogida de información sobre el caso y otra dirigida a responder a las necesidades de vigilancia de la salud colectiva que incluye un conjunto mínimo de datos sobre los aspectos claves para re-organizar los planes de actuación preventiva.

La conducción de la investigación se facilita a través de algoritmos que sintetizan el procedimiento de investigación en tres procesos: (i) el inicio de la investigación, (ii) la recogida de información y (iii) la emisión de informes.

Este procedimiento incorpora dos elementos de ayuda que contribuyen a resolver las incertidumbres que pueden presentarse a los expertos responsables de la investigación en lo que se refiere a la verificación de la exposición potencialmente relacionada con la enfermedad, y el espacio temporal al que ha de retrotraerse la investigación del agente potencialmente causante de la enfermedad.

Para la elaboración de los elementos a verificar se han tenido en cuenta las características específicas de la cadena epidemiológica para cada uno de los seis grupos de enfermedades profesionales contenidos en el anexo I del RD 1299/2006 6 , diferenciando la cadena epidemiológica para agentes químicos, agentes físicos que actúan por exposición, agentes físicos que actúan por mecanismos bio-mecánicos y agentes biológicos. Para cada uno de estos grupos se establecen los elementos a verificar en relación con la exposición o condición de trabajo.

La determinación del espacio temporal sobre el que indagar la exposición está determinado por los periodos de latencia de la enfermedad, un aspecto no resuelto epidemiológicamente para la mayor parte de las enfermedades profesionales y que debe interpretarse siempre de forma orientativa, en este sentido el procedimiento aporta una matriz de periodos orientativos de inducción y latencia que para cada enfermedad o síndrome incluye el o los agentes relacionados etiológicamente con cada enfermedad o síndrome y las recomendaciones en relación al periodo de tiempo más adecuado para indagar la exposición. Estas recomendaciones proceden de diferentes fuentes, principalmente de la Guía de Ayuda para la Valoración de las Enfermedades Profesionales del Instituto Nacional de Seguridad Social y los protocolos de la Red Nacional de Vigilancia Epidemiológica del Instituto de Salud Carlos III. Adicionalmente y según el tipo de enfermedad se han utilizado como referentes las Directrices para la decisión clínica en enfermedades profesionales, INSST- ISCIII, Guías Fisterra para Atención Primaria y Guías de la Sociedad Española de Neumología y Cirugía Torácica (SEPAR).

La búsqueda de la causalidad es el principal valor añadido del procedimiento de investigación. Para la reconstrucción del complejo causal de la enfermedad la Epidemiología Laboral de Campo, debe añadir a las dimensiones epidemiológicas definidas por Hirsch: "Persona", "Tiempo" y "Lugar", una nueva dimensión que exprese y de respuesta a la relación del caso con los procesos de trabajo es decir su "Vinculación Tecnológica".

El complejo causal se construye en base a 6 grupos potenciales de causas que reproducen la cadena epidemiológica para cada uno de los 6 grupos de enfermedades profesionales del anexo del RD mencionado anteriormente: agente, tipo de producto o material, hechos relacionados con la fuente de contaminación, infección o requerimientos biomecánicos, forma de contacto, contagio, exposición o forma de sobrecarga, causas relacionadas con la prevención de riesgos laborales y factores personales/ individuales.

Este procedimiento de investigación de casos de enfermedades profesionales (disponible en https://www.insst.es/-/procedimiento-de-investigacion-de-casos-de-enfermedades-profesionalesano-2019-) basa su fortaleza en los resultados de un ensayo de campo y su consistencia en un alto nivel de acuerdo entre los distintos actores que intervienen en el manejo de la enfermedad profesional.

\section{AGRADECIMIENTOS}

Rafael García Matos, María José Aguado Benedí, Raimundo Aragón Bombín, Carlos Arranz Cordero, Carmen Escalada López, Adrián González Martín, Francisco Marqués Marqués, Rafael Panadés Valls, Miguel Ángel Sánchez de la Arena, Jiri Tvrdy Moix, Marta Zimmermann Verdejo. 


\section{BIBLIOGRAFIA}

1. Oshwiki [sede Web]. Bilbao: EU-OSHA; 2017 [acceso 19 de julio de 2019]. Jukka Takala. Eliminating occupational cancer in Europe and globally. Disponible en: https://oshwiki.eu/wiki/Eliminating_occupational_cancer_in_Europe_and_globally.

2. Grupo de Trabajo sobre profesionales sanitarios de la Comisión Nacional de Seguridad y Salud en el Trabajo. Estudio de la dotación e integración de profesionales sanitarios en los servicios de prevención [Internet]. Madrid: Instituto Nacional de Seguridad y Salud en el Trabajo (INSST); 2001 [2001 ene 31; acceso 19 de julio de 2019]. Disponible en: https://www.insst.es/InshtWeb/Contenidos/Instituto/Comision/GruposTrabajo/prof_san.pdf.

3. Instituto Nacional de Seguridad e Higiene en el Trabajo. Estrategia Española de Seguridad y Salud en el Trabajo 2015-2020. Madrid: Instituto Nacional de Seguridad y Salud en el Trabajo (INSST); 2015.

4. Roel Valdés JM, Maqueda Blasco J. Ensayo de campo de un procedimiento de investigación de casos de enfermedades de origen laboral. Med. segur. trab. [Internet]. 2013 jun [citado 17 jul 2019]; 59 (231):227-34. Disponible en: http:// scielo.isciii.es/scielo.php?script=sci_arttext\& pid=S0465546X2013000200004 $\& \operatorname{lng}=$ es. http://dx.doi.org/10.4321/S0465-546X2013000200004.

5. Instituto Nacional de Seguridad y Salud en el Trabajo. Procedimiento de Investigación de casos de enfermedades profesionales [Internet]. Madrid: Instituto Nacional de Seguridad y Salud en el Trabajo (INSST); 2019 [2019 mar 15; acceso 19 de julio de 2019]. Disponible en: https://www.insst.es/documents/94886/599872/Procedimiento+de+investigacion+de+casos+de+EP.pdf/ d4cf188e-c605-4901-84f1-eec94a2a103e.

6. Real Decreto 1299/2006 de 10 de noviembre de 2006 por el que se aprueba el cuadro de enfermedades profesionales en el sistema de la Seguridad Social y se establecen criterios para su notificación y registro. Boletín Oficial del Estado, nº 302 (19 de diciembre de 2006).

7. Irala J, López C, Carlos S. Introducción. En: Jokin de Irala, Miguel Ángel Martínez-González, María Seguí-Gómez, editores. Epidemiología Aplicada. Bar celona, Ariel S.A; 2008.

8. Maqueda Blasco J. Tipificación del comportamiento epidemiológico de brotes en entornos laborales [tesis doctoral]. Alcalá de Henares: Departamento de Cirugía, Ciencias Médicas y Sociales, Universidad de Alcalá de Henares; 2016. 